\title{
Hidden symmetry and magnetospectroscopy of quantum wells near filling factor $v=2$
}

\section{Citation}

Rashba, E. I., and M. D. Sturge. 2000. “ Hidden Symmetry and Magnetospectroscopy of Quantum Wells Near Filling Factor v = 2." Physical Review B 63 (4) (December 27). doi:10.1103/ physrevb.63.045305.

\section{Published Version}

doi:10.1103/PhysRevB.63.045305

\section{Permanent link}

http://nrs.harvard.edu/urn-3:HUL.InstRepos:25872796

\section{Terms of Use}

This article was downloaded from Harvard University's DASH repository, and is made available under the terms and conditions applicable to Other Posted Material, as set forth at http:// nrs.harvard.edu/urn-3:HUL.InstRepos:dash.current.terms-of-use\#LAA

\section{Share Your Story}

The Harvard community has made this article openly available.

Please share how this access benefits you. Submit a story.

Accessibility 


\title{
Hidden symmetry and magnetospectroscopy of quantum wells near filling factor $\nu=2$
}

\author{
E. I. Rashba* and M. D. Sturge \\ Physics Department, Dartmouth College, Hanover, New Hampshire 03755 \\ (Received 10 February 2000; revised manuscript received 21 July 2000; published 27 December 2000)
}

\begin{abstract}
The magnetoluminescence spectra of symmetric quantum wells containing an electron gas show an abrupt changeover from Landau-level behavior (i.e., linear shift of energy with magnetic field) to quadratic (excitonlike) behavior as the field is increased. This so-called "Mott transition" occurs when the electron filling factor $\nu_{e}$ is 2, i.e., when the lowest Landau level is just filled. We show that the changeover is a natural consequence of the hidden symmetry that has been shown to hold in two-dimensional systems at high fields. This symmetry is broken when the integer parts of $\nu_{e} / 2$ and $\nu_{h} / 2$ differ $\left(\nu_{h}\right.$, the hole filling factor, is very small in these experiments). This symmetry breaking can also account for the new emission bands that are observed in such spectra when $\nu_{e}$ becomes larger than 2 , and these bands can be described, at least qualitatively, in the framework of the magnetoexciton theory. These bands include the red-shifted magnetoplasmon and shake-up satellites and the blue-shifted cyclotron satellites. We also discuss the possible existence of a new type of trion (charged exciton), associated with the electron $n_{e}=1$ Landau level, and conclude that these trions should be observable.
\end{abstract}

DOI: 10.1103/PhysRevB.63.045305

PACS number(s): 78.66.Fd, 71.35.Ji, 71.30. $+\mathrm{h}$

\section{INTRODUCTION}

The low-temperature photoluminescence spectrum of a quantum well $(\mathrm{QW})$ containing a high density $\left(\gtrsim 10^{11} \mathrm{~cm}^{-2}\right)$ of electrons is a broad band with a width (after correction for inhomogeneous broadening) close to the Fermi energy. When a magnetic field $B$ is applied, this band breaks up into relatively sharp transitions between Landau levels (LL's) of holes and electrons. ${ }^{1}$ The LL energy measured from the band bottom is $\left(n_{e}+1 / 2, n_{h}+1 / 2\right) \hbar \omega_{c}$, where $\omega_{c}$ is the appropriate cyclotron frequency, and $n_{e}, n_{h}=0,1,2 \ldots$ (for allowed transitions $n_{e}=n_{h}$ ). The transition energy varies nearly linearly with $B$, as expected theoretically if the Coulomb interaction of the electrons is neglected. However, for symmetric QW's the $B$ dependence of the lowest transition (i.e., the $0-0$ LL transition) changes abruptly, at a certain critical field, to approximately quadratic. As is seen in Fig. 1, for fields above this critical value, the emission energy is very close to the transition from the singlet negative trion state observed in the same QW at low electron density (a negative trion is a negatively charged exciton, the excitonic analog of the $\mathrm{H}^{-}$ ion $)^{2-8}$ and shows almost the same $B$ dependence as that of the exciton. As is seen in Fig. 1, the emission spectra show another remarkable regularity: the optical transition energy at fields above the critical value shows almost the same B dependence as that of the exciton. This changeover from linear (LL-like) to quadratic (trionlike) behavior is not observed in asymmetric QW's.

The spectrum changes in other ways as well: the linewidth decreases and the line profile changes, as shown in Fig. 2. Cyclotron satellites blue shifted from the $0-0$ line by approximately $\hbar \omega_{c}$, which are prominent at low field, disappear as abruptly as the nonuniformity of the sample permits; and a red-shifted satellite, which is only a shoulder in Fig. 2 but is well resolved in the spectra of Finkelstein et al., ${ }^{9}$ also disappears. These authors also reported that shake-up processes, which produce satellites red shifted from the $0-0$ transition line by one or two cyclotron quanta, sud- denly become very weak. There is also a change in the radiative decay time (see Fig. 3 of Ref. 7).

The interesting thing about this critical field is that, regardless of the electron density, it is always the field at which the lowest electron LL is just filled; i.e., the field at which the electron filling factor $\nu_{e}=2$, as shown in Fig. 4 of Ref. 7 (note that $\nu_{e}$ is proportional to electron density and inversely proportional to $B$ ). In this paper we argue that this is not a coincidence, but is a consequence of "hidden symmetry" (HS) inherent in two-dimensional (2D) electron systems in strong magnetic fields. ${ }^{10-15}$ The mechanism driving this changeover has nothing to do with that driving the true metal-insulator Mott transition, and it would be better named a "symmetry-driven transition." This point is discussed more fully in Ref. 16.

Our analysis of the experimental results is based on data obtained on GaAs QW's by several independent experimental groups $;{ }^{1,5-7}$ some of these data were summarized in Ref. 7. Similar results have been obtained in CdTe QW's., ${ }^{2,17}$ Our theoretical analysis is based on a consistent application of the hidden symmetry concept and of the magnetoexciton formalism to the optical spectra near $\nu_{e}=2$; the term magnetoexciton (ME) is used for an exciton in a strong magnetic field. The main goals of this paper are to show that the phenomena listed above are mutually connected, to discuss this connection, and to establish physical mechanisms underlying these phenomena. The assignment of red- and blue-shifted satellites that we propose supports our view that the " $v_{e}=2$ transition" is due to breaking the HS and provides a coherent description of the emission spectra for $\nu_{e}$ slightly larger than 2. We do not aim to obtain precise numerical fits to the experimental data, but rather to achieve a semiquantitative understanding of the observations in terms of simple physical concepts. Nevertheless, our estimates (based on the ME concept) of the positions of red-shifted magnetoplasmon satellites and of the dependence of the intensity of blue-shifted cyclotron satellites on the concentration of free electrons are in quite reasonable agreement with experimental data. It is 


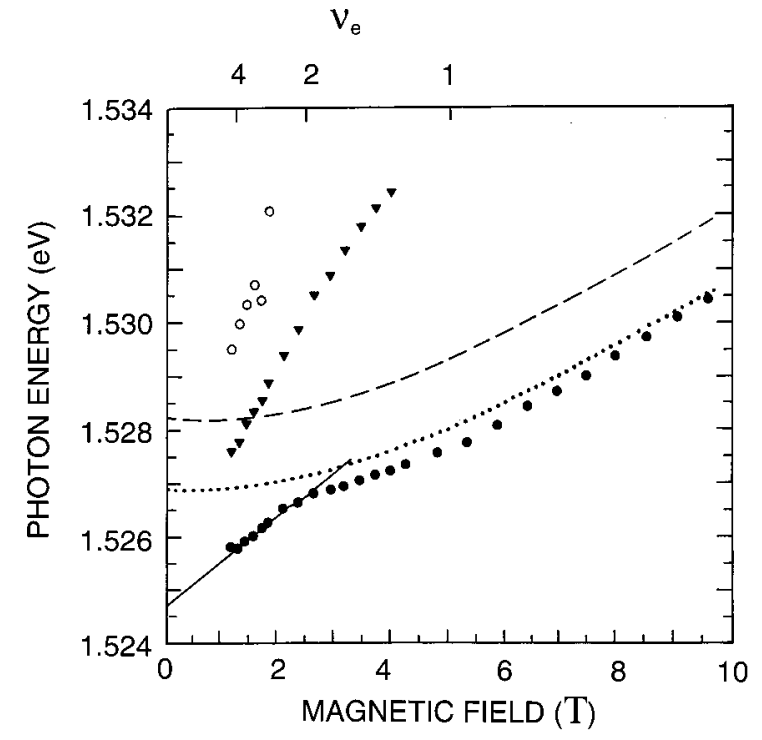

FIG. 1. Peak positions of the photoluminescence spectrum of a symmetric $200 \AA$ quantum well containing approximately 1.2 $\times 10^{11}$ electrons $/ \mathrm{cm}^{2}$ at $4.2 \mathrm{~K}$, as a function of applied magnetic field (adapted from Ref. 7). The filling factor at different fields is shown along the top of the figure (note that because of the uncertainty in the electron density, the absolute value of $\nu_{e}$ is uncertain to $\pm 20 \%$ ). The filled circles are the positions of peaks that were assigned in Ref. 7 to the $0-0$ transition, the triangles to $1-1$, and the open circles to 2-2 (in this paper we propose new assignments of the latter two peaks-see Sec. V). The "2-2"' peak disappears, and the " $1-1$ " peak becomes very weak, when $\nu_{e}$ drops to about 2 (see Fig. 2). The full line is the best fit to the linear shift of the $0-0$ peak of the lowest Landau level at low fields, and has a slope $0.8 \mathrm{meV} / \mathrm{T}$, about $80 \%$ of the slope expected from the reduced mass of the electron and hole. The dashed line is the observed field dependence of the exciton peak at low electron density, and the dotted line that of the singlet trion.

remarkable that in spite of the fact that the concepts of HS and ME's can be strictly justified only in the high-magnetic field limit, we find that they work well and provide a reliable classification of elementary excitations even at the moderate fields (typically a few tesla) used in most of the experimental studies.

The plan of the paper is as follows. In Sec. II we present the basic concepts of HS and ME's and mention some experimental results that confirm the applicability of these concepts. In Sec. III we argue that the behavior of the emission spectra of symmetric QW's in the $\nu_{e}<2$ region clearly demonstrates the dominating role of the HS. In Secs. IV and V, two specific mechanisms of breaking HS are discussed in terms of ME's. These lead to the appearance of new emission bands (red-shifted magnetoplasmon satellites and blueshifted cyclotron satellites due to scattering of ME's by free electrons) which enable us to compare the predictions of our theory with experimental data. In Sec. VI we argue that a new type of trion exists for $\nu_{e}>2$; the details of the mathematical arguments for its existence are given in the Appendix. In Sec. VII we briefly discuss, by analogy with molecular excitons, the problem of magnetoexciton band splitting

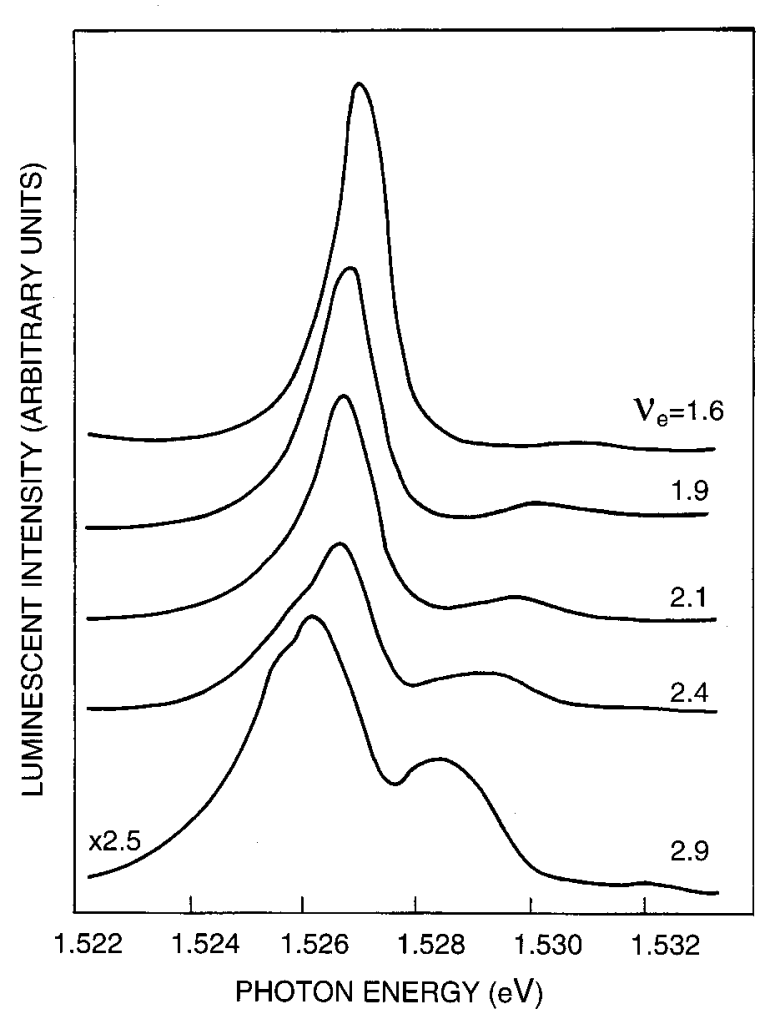

FIG. 2. Photoluminescence spectrum of the same quantum well as in Fig. 1, in the vicinity of $\nu_{e}=2$ (from Ref. 16). Note the disappearance of the cyclotron satellite on the high-energy side of the line, and of the magnetoplasmon shoulder on the low-energy side, when $\nu_{e}$ falls below 2 .

and intensity borrowing in $\nu_{e}<2$ region, and in Sec. VIII we summarize our conclusions.

\section{HIDDEN SYMMETRY AND MAGNETOEXCITONS}

The 2D many-particle problem in a finite magnetic field $B$ is too complicated for rigorous theoretical analysis. Even the two-particle problem, the exciton, can be solved exactly only in two limits: $B=0$ and $B=\infty$. Fortunately, in the $B=\infty$ limit there exists a class of many-particle $2 \mathrm{D}$ systems that possesses hidden symmetry: this allows one to find their exciton spectra without solving the many-particle problem.

The basic conditions for HS are (i) charge-symmetric interaction of electrons and holes, (ii) all carriers are in the lowest LL, and (iii) strict $B=\infty$ and 2D limits. ${ }^{10-14}$ A more general condition for HS to hold is that the filling factors for electrons and holes, $\nu_{e}$ and $\nu_{h}$, obey the condition $2(N$ $+1) \geqslant \nu_{e}, \nu_{h} \geqslant 2 N$, where $N \geqslant 0$ is an integer. ${ }^{15}$ Under these conditions, even when the filling factors are nonintegral, the frequency of an optical transition creating or destroying a magnetoexciton is exactly equal to the frequency of this transition for integer filling factors, $\nu_{e}=\nu_{h}=2 N .{ }^{18}$ In the case $N=0$, this implies the remarkable result that the frequency of the optical transition is unaffected by the presence of free electrons or holes and the transition remains narrow, so long as both filling factors are less than 2. This statement can be proven either by operator algebra ${ }^{13-15}$ or by mapping 
of the electron-hole system onto an auxiliary spin-1/2 electron system. ${ }^{12}$

ME's produced by light have momentum $\mathbf{k}=0$. Under the conditions of HS, the many-particle electronic wave function of a crystal containing a $k=0 \mathrm{ME}$ is a product of the wave function of the electronic background and the wave function of a $k=0 \mathrm{ME}$ in an empty crystal. However, the multiplicative structure of the wave function and absence of any level shift of the $k=0$ ME does not mean that ME's with $k \approx 0$ do not interact with the background. This interaction is in fact rather strong. ${ }^{14}$ However, HS makes it impossible to investigate electron-electron $(e-e)$ interaction at high-magnetic field by optical experiments. Some of the consequences of the violation of HS have been investigated in a number of theoretical papers. ${ }^{19-23}$

In particular, HS excludes the existence of trion bands in absorption or emission spectra in the $B \rightarrow \infty$ limit. In fact, in this limit the singlet trion (total spin of two electrons $S$ $=0)$ becomes unbound, while the transition to the triplet trion $(S=1)$ becomes forbidden. ${ }^{22,24}$ For moderate magnetic fields $B \leqq 10 \mathrm{~T}$, however, the binding energy of the singlet trion is larger than that of the triplet. ${ }^{25-27}$ In particular, the data indicated by the dotted line in Fig. 1 are for negative singlet trions. As pointed out above, HS provides a connection between optical spectra at fractional and integer values of $\nu_{e}$ and $\nu_{h}$. Magnetoexciton theory gives the ME dispersion law, the position of the exciton transition and its intensity for arbitrary $n_{e}$ and $n_{h}$, so long as they are at the Fermi level and $\nu_{e}$ and $\nu_{h}$ are close to integers (see Ref. 28 and references therein). Such ME's are stable against intraband decay. The theory of ME's obeys the HS requirements and, for strong enough magnetic fields, takes the Coulomb interaction into account consistently.

A convenient dimensionless measure of the magnetic field strength is $\gamma=\hbar \omega_{c} / 2 \mathrm{Ry}$, where $\mathrm{Ry}=m e^{4} / 2 \epsilon^{2} \hbar^{2}$ is the 3D Rydberg, $m$ is reduced mass $m=m_{e} m_{h} /\left(m_{e}+m_{h}\right)$, and $\omega_{c}$ $=e B / m c$. It is difficult to evaluate, theoretically, the accuracy of the specific predictions of HS and ME theory when $\gamma$ is finite. Numerical data for symmetric QW's (Ref. 25) show that singlet trions exist over the whole range of $\gamma$ values and QW widths of practical interest, showing that the HS does not strictly hold for them. On the other hand, as we shall see, available experimental data indicate that some of the predictions relevant to our problem hold down to quite low values of $\gamma$.

For example, Butov et al. ${ }^{29}$ have shown that for $\gamma \approx 1.5$ the positions of exciton transitions for a neutral magnetoplasma in symmetric QW's with integer fillings $\nu_{e} \approx \nu_{h} \approx 2$, 4 , and 6 are in agreement with the prediction of the ME theory for all stable ME states. Furthermore, the positions of the transitions to and from these states do not change when the filling factors vary between two consecutive even integers, ${ }^{30}$ in agreement with the predictions of HS. On the other hand, states remote from the Fermi level are unstable and do not obey the HS predictions. For these states, the accuracy of the ME approach is much lower, and the theory provides only a qualitative description of basic regularities.

Another example of the applicability of HS at moderate $\gamma$ has been reported by Finkelstein et al., ${ }^{9}$ who studied shake-up processes in QW's containing a unipolar electron gas, in which $\nu_{h} \sim 0$ while $\nu_{e}$ varies from a high value to less than 2. Shake-up processes produce satellites red shifted from the 0-0 transition line by one or two cyclotron quanta. These authors found that the probability of shake-up processes decreases drastically when $\nu_{e}$ falls below 2 , and they related this decrease to the fact that these processes are forbidden by HS when $\nu_{e}<2$. Under the same conditions, they have also observed a feature denoted as $\mathrm{SU}_{0}$ (see Fig. 3 in Ref. 9) which shows the same decrease in probability for $\nu_{e}<2$. This feature is discussed in more detail below in Sec. IV.

According to ME theory, ${ }^{19,31-33}$ each pair of LL's generates a single branch of ME's that are completely described by the $2 \mathrm{D}$ momentum $\mathbf{k}$. In the notation of Ref. 28 , the creation operators of ME's are

$$
\begin{aligned}
\mathcal{A}_{\alpha m, \beta n}^{\dagger}(\mathbf{k}, \sigma \tau)= & (2 \pi / S)^{1 / 2} \sum_{p} a_{\alpha m \sigma}^{\dagger}\left(p-k_{y} / 2\right) a_{\beta n \tau} \\
& \times\left(p+k_{y} / 2\right) \exp \left(i k_{x} p\right) .
\end{aligned}
$$

Here, the indices $\alpha$ and $\beta$ stand for the conduction and valence bands $(\alpha, \beta=c, v), m$ and $n$ for Landau levels, and $\sigma$ and $\tau$ for spin states, $p$ is the momentum quantum number in the Landau gauge with the vector potential $\mathbf{A}=(0, x, 0)$, $a_{\beta n \tau}(p)$ are annihilation operators of Landau states, and $S$ is the normalization area. In Eq. (1), and in most of the equations that follow, length is measured in units of the magnetic length $l(B)=(c \hbar / e B)^{1 / 2}$ and momentum in units of $l^{-1}$. The energy of an interband magnetoexciton $(\alpha=c, \beta=v)$ includes the Coulomb electron-hole $(e-h)$ attraction, $\varepsilon_{m n}(\mathbf{k})$, and the exchange energy, $\mathcal{H}_{m n}^{\mathrm{HF}}$, of the $c m \sigma$ electron with the electrons in LL's with quantum numbers $m^{\prime}<m$, and a similar contribution for the $v n \tau$ hole. Intraband excitons $\alpha=\beta$ $=c$ are usually termed magnetoplasmons (MP's). Their energies include an additional term related to the resonance excitation transfer $H_{\mathrm{r}}$. When $n$ is an even integer, $H_{\mathrm{r}}$ generates two branches of the MP spectrum: charge and spin MP's.

When $\nu_{e}<2$ and photoholes are in thermal equilibrium in the lowest LL, $n_{h}=0$, HS requires that the emission spectrum consist of a single narrow line whose position is exactly the same as for $(0,0)$ ME's in the empty crystal, $E_{\mathrm{X}}(\mathbf{k}=0)$ $=-\sqrt{\pi / 2} E_{\mathrm{C}} \approx-1.25 E_{\mathrm{C}}$, which is the bottom of the lowest ME band. $E_{\mathrm{C}}(B)=e^{2} / \epsilon l$ is the magneto-Coulomb energy typical of ME's. Here and below we write out only the Coulomb contributions to the energy and omit $E_{\mathrm{G}}$, the cyclotron energy, and the Zeeman energy, which either cancel out from final results for the relative positions of the $0-0$ transition and its MP satellites, or can be easily added to them when needed. Following the conventional notation, we designate the $0-0 \mathrm{ME}$ as $X$.

\section{SYMMETRY-DRIVEN TRANSITION}

Let us consider in more detail the most striking features of Fig. 1. The electron concentration here is $N_{e}=1.2 \times 10^{11}$ $\mathrm{cm}^{-2}$. Nevertheless, for $\nu_{e}<2$ the position of the emission 
band as a function of $B$ follows the position of the singlet trion emission band in the empty crystal $N_{e}=0$ within less than $0.5 \mathrm{meV}$. This cannot be attributed to independence of the trion quantum state of the surrounding electrons. Indeed, since trions are loosely bound entities with the binding energy an order of magnitude less than that of an exciton, they should be especially sensitive to changing $\nu_{e}$. Therefore, there must be some special reason why the position of the emission band is not sensitive to such a drastic change of the trion wave function. The fact that the "trionlike" emission band shows nearly the same $B$ dependence as the exciton and is only about $1 \mathrm{meV}$ lower, suggests strongly that the HS discussed in Sec. II is the underlying mechanism. The fact that the changeover from "LL-like" to "trionlike" dependence on $B$ is at $\nu_{e}=2$ for specimens with widely different $N_{e},{ }^{7}$ supports this suggestion. While some violation of the HS manifests itself in the very fact that a singlet trion exists, the small magnitude of the trion level shift from the exciton indicates that the violation is minor.

The changes in the shape of the emission spectrum near $\nu_{e}=2$ shown in Fig. 2 also find a natural explanation in the framework of the HS concept. For $\nu_{e}<2$, HS operates and, therefore, only the $0-0 \mathrm{ME}$ transition is allowed. Hence, the emission band is narrow as is observed. For $\nu_{e}>2$, the HS is violated, and transitions between LL's characteristic of an electron gas dominate the emission. Electron-electron interaction manifests itself in the spectra both by shifting the principal peaks and producing satellite bands. Specific mechanisms of developing different sidebands for $\nu_{e}$ slightly larger than 2 are discussed below in Secs. V and VI.

Independent evidence that the $\nu_{e}=2$ changeover is due to a change in the dynamic symmetry of the system comes from the comparison of the emission spectra of symmetric and asymmetric QW's. In an asymmetric QW, the vertical electric field separates the electrons and holes, breaking the electron-hole equivalence that is a condition for HS to hold. Thus, our interpretation in terms of HS would predict that there should be no changeover to trion-like (or excitonlike) behavior at $\nu_{e}=2$ in an asymmetric well. If, on the other hand, the changeover were due to the shrinkage of the exciton wave function by the magnetic field, which reduces the effects of screening and band filling (i.e., a so-called "Mott" or "metal-insulator" transition), no difference between symmetric and asymmetric wells would be expected. Our prediction is borne out by experiment. For example, there is no changeover in the spectrum of a single-side modulation doped QW reported by Manfra et al. ${ }^{34}$ (Fig. 3 of their paper), in which the principal emission peak shows nearly linear (i.e., LL-like) dependence on $B$ throughout the $\nu_{e}<2$ region. This is even more strikingly illustrated by the data of Hayne et al. ${ }^{35}$ who observed the $\nu_{e}=2$ changeover in the spectra of a single asymmetric QW only under strong illumination, and attributed the change to the flattening of the bands. Such flattening makes the QW nearly symmetric.

Let us mention in conclusion that one might expect changes in the emission spectra in the vicinity of $\nu_{e}=2$, because at this filling factor a gap opens at the Fermi level, changing the screening of the Coulomb interaction. However, the gap disappears again as $\nu_{e}$ is reduced below 2, so that this mechanism provides no explanation for the continued persistence of the trionlike spectrum at fields above the critical value. The changeover is symmetry driven and has also nothing to do with the field-induced metal-insulator Mott transition mentioned in the previous paragraph.

In what follows, we analyze the spectra in terms of HS and apply the ME formalism. Such an approach might seem inconsistent, since the $\nu_{e}=2$ transition has been observed in Ref. 7 at $\gamma \approx 1 / 2$ and in Ref. 5 even at $\gamma \approx 1 / 4$. However, the experimental fact that it is the filling factor $\nu_{e}=2$ that is found to be significant for the transition, regardless of $\gamma$, and the results of Refs. 29 and 30, indicate that the quantum numbers that describe the electron-hole system for $\gamma \gg 1$ may still provide a satisfactory classification of quantum states for moderate values of $\gamma$. It is one of the goals of this paper to see how useful the HS and the ME approach can be in interpreting optical spectra at moderate and even low $\gamma$.

\section{MAGNETOPLASMON-ASSISTED RECOMBINATION}

We will discuss the channels of radiative recombination that open for $\nu_{e}>2$, taking into account the fact that direct recombination of an $n_{e}=1$ electron with a $n_{h}=0$ hole is forbidden. We will not consider the red-shifted "shake-up" cyclotron satellites already discussed in Ref. 9.

The first new recombination channel is MP-assisted annihilation of ME $(1,0)$. This is a radiative recombination of a 0-0 $e$ - $h$ pair, leaving an $n_{e}=0$ Fermi hole to which the $n_{e}$ $=1$ electron binds, forming MP $(1,0)$. For $0<\nu_{e}-2 \ll 1$, the interaction between $n_{e}=1$ electrons can be neglected and a straightforward calculation shows that the matrix element for this process is ${ }^{36}$

$$
\begin{aligned}
& \left\langle\mathrm{v}\left|\mathcal{A}_{c 1, c 0}\left(\mathbf{k}^{\prime}, \sigma_{e}^{\prime} \sigma_{\mathrm{Fh}}\right) H_{\mathrm{opt}} \mathcal{A}_{c 1, v 0}^{\dagger}\left(\mathbf{k}, \sigma_{e} \sigma_{h}\right)\right| \mathrm{v}\right\rangle \\
& \quad=\delta_{\sigma_{e}^{\prime} \sigma_{e}} \delta_{\sigma_{\mathrm{Fh}} \sigma_{h}} \delta_{\mathbf{k}^{\prime} \mathbf{k}} .
\end{aligned}
$$

Here $|v\rangle$ is the vacuum state in which the valence band and the $n_{e}=0$ Landau level of the conduction band are completely filled, while the $n_{e}>0$ LL's are empty, and

$$
H_{\mathrm{opt}}=\sum_{p n \sigma}\left\{a_{\mathrm{c} n \sigma}(p) a_{\mathrm{v} n \sigma}^{\dagger}(p)+a_{\mathrm{v} n \sigma}(p) a_{\mathrm{c} n \sigma}^{\dagger}(p)\right\}
$$

is the standard optical transition operator expressed in the units of the band-to-band transition matrix element [note that the matrix element (2) of the MP-assisted transition does not depend on either the electron and hole spins, $\sigma_{e}$ and $\sigma_{h}$, of the initial ME or on the strength of the Coulomb interaction]. In the same units, the matrix element of the radiative annihilation of a ME $(0,0)$ with momentum $\mathbf{k}=0$ is $\sqrt{S / 2 \pi l^{2}}$. The meaning of this relation becomes clear if we use Eq. (3) to obtain the oscillator strengths of the inverse processes in the absorption spectrum: we find $f_{0-0}=f_{1,0}$. Here $f_{0-0}$ is the oscillator strength of the $0-0$ transition per area $2 \pi l^{2}$ in an empty crystal, while $f_{1,0}$ is the oscillator strength for radiative transformation of a MP $(1,0)$ into $\mathrm{ME}(1,0)$, per magnetoplasmon present in the initial state. ${ }^{37}$

The MP-assisted transition from the ME $(1,0)$ state produces MP satellites of the 0-0 band, whose positions depend 
on the dispersion laws of the $(1,0)$ magnetoexciton and of the MP's. The ME dispersion law found by the standard procedure of Ref. 28 is

$$
\begin{aligned}
E_{1,0}^{\mathrm{ME}}(k)= & -\frac{1}{2} \sqrt{\frac{\pi}{2}}\left\{1+\mathrm{I}_{0}\left(\frac{k^{2}}{4}\right) \exp \left(-\frac{k^{2}}{4}\right)\right. \\
& \left.+\frac{k^{2}}{2}\left[\mathrm{I}_{0}\left(\frac{k^{2}}{4}\right)-\mathrm{I}_{1}\left(\frac{k^{2}}{4}\right)\right] \exp \left(-\frac{k^{2}}{4}\right)\right\} E_{\mathrm{C}},
\end{aligned}
$$

where $\mathrm{I}_{\mathrm{n}}(x)$ are modified Bessel functions. Equation (4) equals $-\sqrt{\pi / 2} E_{\mathrm{C}} \approx-1.25 E_{\mathrm{C}}$ at $k=0$ and has a flat minimum $E_{1,0}^{\mathrm{ME}}\left(k_{\text {min }}\right) \approx-1.35 E_{\mathrm{C}}$ for $k_{\min } \approx 1.19$. It is remarkable that $E_{1,0}^{\mathrm{ME}}\left(k_{\text {min }}\right)<E_{\mathrm{X}}(k=0)$ despite the fact that the binding energy of $X$ is nearly twice as large as that of ME $(1,0)$. The reason is that exchange interaction with $n_{e}=0$ electrons, $\mathcal{H}_{1,0}^{\mathrm{HF}}=-\sqrt{\pi / 8} E_{\mathrm{C}}$, lowers the energy of $\operatorname{ME}(1,0)$.

For $\nu_{e}=2$, there are two branches of the MP spectrum, corresponding to charge and spin excitations, both MP's having equal coupling constants to $(1,0)$ ME's. The dispersion laws of these MP's are shown in Fig. 4(a) of Ref. 32. For $k_{\mathrm{B}} T \ll E_{\mathrm{C}}$, one expects a single MP satellite to originate from each branch of the MP spectrum, the momenta of both MP's being $\approx k_{\text {min }}$ and their energies $E_{\mathrm{MP}}^{\mathrm{ch}}\left(k_{\min }\right) \approx 0.49 E_{\mathrm{C}}$ and $E_{\mathrm{MP}}^{\mathrm{sp}}\left(k_{\min }\right) \approx-0.09 E_{\mathrm{C}}$, respectively. Both energies are reckoned from the electron cyclotron energy $\hbar \omega_{e}$, which is the energy of $k=0$ MP's from Kohn's theorem. ${ }^{38}$ With $E_{\mathrm{X}}(k$ $=0) \approx-1.25 E_{\mathrm{C}}$ and $E_{1,0}^{\mathrm{ME}}\left(k_{\min }\right) \approx-1.35 E_{\mathrm{C}}$, the charge-MP satellite is red shifted as compared to the $0-0 \mathrm{ME}$ transition by $\approx 0.6 E_{\mathrm{C}}$, while the spin-MP satellite merges with it. Because the ME $(1,0)$ and MP $(1,0)$ dispersion curves, and their differences, reach extremal values at $k \neq 0$, the combined density of states shows 1D van Hove singularities. Therefore, as temperature increases, the satellites can acquire peculiar spectral shapes with spikes at their edges.

A well-resolved satellite has been observed at $1.5 \mathrm{~K}$ and $\gamma \approx 1.5$ by Finkelstein et al. ${ }^{9}$ who denoted it " $\mathrm{SU}_{0}$ " (see their Fig. 3). Its red shift $\approx 0.5 E_{\mathrm{C}}$ is in satisfactory agreement with the estimate for the charge-MP satellite discussed above. There is also a strong low-frequency shoulder on the $0-0$ band which might be the spin-MP satellite. The assignment of the $\mathrm{SU}_{0}$ band proposed in Ref. 9 has a certain similarity to ours. However, they describe this transition as a shake-up process with some resonance denominator while, as can be seen from Eq. (2), it occurs in the zeroth order in the Coulomb interaction, which is why it is so strong. ${ }^{39} \mathrm{Be}-$ cause the minimum of $E_{1,0}^{\mathrm{ME}}(k)$ is flat and shallow, only about $0.1 E_{\mathrm{C}}$, the width of the MP satellite should increase to the width of the charge-MP spectrum $\left(\approx 0.4 E_{\mathrm{C}}\right)$ at rather low temperature (about $10 \mathrm{~K}$ at $9 \mathrm{~T}$ ). In fact, a strong $T$ dependence of the shape of $\mathrm{SU}_{0}$ satellite at $4.2 \mathrm{~K} \gtrsim k_{\mathrm{B}} T$ was reported in Ref. 9, but the above explanation of it is only tentative. For a related but different scenario see Sec. VI below.

The low-frequency shoulder of the trion emission band observed at $4.2 \mathrm{~K}$ in Ref. 16, and seen in Fig. 2, appears to be due to the same cause. As $B$ decreases it appears at $\nu_{e}$ $\approx 2$ and grows rapidly with growing $\nu_{e}$. It is red shifted by $\approx 0.2 E_{\mathrm{C}}$ from the $\operatorname{ME}(0,0)$ transition. It seems natural to assign it as a charge-MP satellite, and the deviation of the redshift from the theoretical estimate is perhaps not surprising, taking into account that $\gamma$ is $\approx 0.5$. $^{40}$

As we pointed out in Sec. II, the ME approach can be strictly justified only in the strong magnetic field limit, $\gamma$ $\gg 1$. In this section, we have applied the ME theory to obtain the relative positions of different bands in units of $E_{\mathrm{C}}$, and compared these positions with experimental data taken for $\gamma \approx 1$, and even for $\gamma$ less than 1 . We found reasonable agreement between the theory and experiment, but it must be remembered that the absolute positions of exciton bands in this range of $\gamma$ cannot be expressed in terms of $E_{C}$. In fact the bands still show the nearly quadratic in $B$ behavior characteristic of the weak $B$ regime.

The fact that red-shifted MP satellites appear when $\nu_{e}$ increases through 2 supports the view that the change in behavior of the emission experimentally observed at $\nu_{e}=2$ is a manifestation of a changeover from the "HS" regime to the "free electron" or "LL" regime. For $\gamma \leq 1 / 2$, the MP satellite is no longer well resolved from the 0-0 transition and appears as a shoulder. This shifts the $0-0$ band to lower energy compared to its HS position and broadens it, as observed in Ref. 7. The shift and broadening increase rapidly with decreasing $B$, as $\nu_{e}$ grows and new $\mathcal{H}_{m n}^{\mathrm{HF}}$ exchange terms appear and new recombination channels open. When the position of this broad band is treated in terms of Landau levels, the Coulomb interaction manifests itself as a considerable increase of the cyclotron mass (by a factor up to 2 in Ref. 5) - a well-known effect in the theory of Coulomb corrections in the metallic phase. ${ }^{41}$ The ME approach is completely inapplicable in this parameter range.

\section{SCATTERING-INDUCED EMISSION}

Another channel of radiative recombination that turns on for $\nu_{e}>2$ is the radiative recombination of $(1,0)$ ME's made possible by elastic scattering by $n_{e}=1$ electrons, whose concentration is $N_{e}^{(1)}=\left(\nu_{e}-2\right) / 2 \pi l^{2}$. Direct radiative recombination of these ME's is forbidden by the $n-n$ selection rule and also by momentum conservation since the bottom of the ME $(1,0)$ band is at $k_{\min } \neq 0$. This transition is about $\hbar \omega_{c}$ above the $0-0$ transition and should compete with the spectrally close recombination of $(1,1)$ ME's, whose concentration is less than that of $(1,0)$ ME's by a factor about $\exp \left(-\hbar \omega_{h} / k_{\mathrm{B}} T\right)$, where $\omega_{h}$ is the hole cyclotron frequency. For $B \approx 2.5 \mathrm{~T}$ and $T \approx 4.2 \mathrm{~K}$ this factor is about 0.07 .

Several channels of the ME-electron scattering contribute to this process. Because of the inequality $m_{e} \ll m_{h}$, we select only the process with the smallest denominator, which is the hole cyclotron energy $\hbar \omega_{h}$. This process involves the virtual excitation of the hole to the $n_{h}=1 \mathrm{LL}$ due to its Coulomb interaction with the electrons, followed by recombination of the hole with an $n_{e}=1$ electron. The probability of this process is controlled by the matrix element

$$
\begin{aligned}
& M\left(p^{\prime} \tau^{\prime} \mid \mathbf{k} \sigma_{e} \sigma_{h}, p \tau\right) \\
& \quad=\left\langle v\left|a_{\mathrm{c} 1 \tau^{\prime}}\left(p^{\prime}\right) H_{\mathrm{opt}} H_{\mathrm{int}} \mathcal{A}_{\mathrm{c} 1, \mathrm{v} 0}^{\dagger}\left(\mathbf{k}, \sigma_{e} \sigma_{h}\right) a_{\mathrm{c} 1 \tau}^{\dagger}(p)\right| \mathrm{v}\right\rangle,
\end{aligned}
$$


where $p^{\prime} \tau^{\prime}$ and $p \tau$ are the electron momentum and spin in the final and initial states, respectively, and $\mathbf{k}, \sigma_{e}$, and $\sigma_{h}$ are the ME $(1,0)$ momentum and its electron and hole spins. $H_{\text {int }}$ is the standard operator of the $e-e$ and $e-h$ interaction. ${ }^{28}$ There are two virtual scattering processes that can contribute to this matrix element: (i) potential scattering of the $\operatorname{ME}(1,0)$ to the $k=0, \operatorname{ME}(1,1)$ state with spins unchanged, and (ii) exchange scattering of the $\operatorname{ME}(1,0)$ to the $k=0, \operatorname{ME}(1,1)$ state with the new spin configuration $\left(\tau, \sigma_{h}\right)$. Both scattering events are followed by radiative annihilation of ME $(1,1)$. Straightforward calculation shows that the matrix element of process (ii) vanishes, while process (i) results in the matrix element:

$$
\begin{aligned}
\left|M\left(p^{\prime} \tau^{\prime} \mid \mathbf{k} \sigma_{e} \sigma_{h}, p \tau\right)\right|= & \delta_{\tau \tau^{\prime}} \delta_{\sigma_{e} \sigma_{h}} \delta_{p^{\prime}, p-k_{y}} \sqrt{\pi l^{2} / S} \\
& \times\left|1-k^{2} l^{2} / 2\right| \exp \left(-k^{2} l^{2} / 2\right) E_{\mathrm{C}},
\end{aligned}
$$

when written in dimensional units. Dividing it by the energy denominator $\hbar \omega_{h}$, squaring the ratio, and summing it over all scatterers (i.e., over all $n_{e}=1$ electrons with different $p, \tau)$, we find for the probability of the scattering induced radiative recombination of an $\operatorname{ME}(1,0)$ with momentum $k$ :

$$
\begin{aligned}
W\left(k, \sigma_{e} \sigma_{h}\right)= & \delta_{\sigma_{e} \sigma_{h}}\left[\left(\nu_{e}-2\right) / 2 \gamma\right]\left(m_{h} / m_{e}\right)^{2} \\
& \times\left(1-k^{2} l^{2} / 2\right)^{2} \exp \left(-k^{2} l^{2}\right) .
\end{aligned}
$$

It is important to note that $W\left(k, \sigma_{e} \sigma_{h}\right)$ includes the large factor $\left(m_{h} / m_{e}\right)^{2}$, which for GaAs is about 20 , so that the mechanism is effective as long as $k l \lesssim 1$. Equation (7) determines the spectral shape of the blue-shifted cyclotron satellite of the $0-0$ transition. Averaging Eq. (7) over a Maxwellian distribution of ME's gives $W(T)$, and multiplying this by the square of the band-to-band transition matrix element gives the absolute intensity of the cyclotron satellite in the emission spectrum. When $\nu_{e}$ increases through $\nu_{e}=2$, this satellite should appear simultaneously in emission and absorption spectra, but with different spectral shapes.

A similar process may also exist if the initial state for emission is a bound state of MP $(1,0)$ and an $n_{e}=1$ electron, i.e., the trion $T(1,1 ; 0)$ discussed in the next section. One can roughly evaluate the probability of the radiative decay of such a trion from Eq. (7). For $\left|E_{1,0}^{\mathrm{T}}\right| \ll E_{\mathrm{C}}$, a trion includes a weakly bound $n_{e}=1$ electron that plays the role of a free electron as a scatterer of the ME $(1,0)$. The effective electron density $\nu_{\text {eff }}$ in a trion, playing the role of the factor $\nu_{e}-2$ in Eq. (7), can be estimated by averaging the electron density $\left|\psi_{\mathrm{el}}(\mathbf{r})\right|^{2}$ over the ME wave function:

$$
\nu_{\mathrm{eff}} / 2 \pi l^{2}=\int d \mathbf{r}\left|\psi_{\mathrm{el}}(\mathbf{r})\right|^{2}\left|\psi_{\mathrm{ME}}(\mathbf{r})\right|^{2}
$$

Here, $\psi_{\mathrm{el}}(\mathbf{r})$ and $\psi_{\mathrm{ME}}(\mathbf{r})$ are wave functions of the electron and the ME bound to it, respectively. One also has to average the $k$-dependent factors in the right-hand side of Eq. (7) over the ME wave function in the momentum representation. Unfortunately, the function $\psi_{\mathrm{ME}}(\mathbf{r})$ cannot be calculated analytically because of the intricate dispersion law of $(1,0)$
ME's, see Eq. (4). Therefore, we estimate the right-hand side of Eq. (8) using $\left|\psi_{\mathrm{ME}}(\mathbf{r})\right|^{2} \sim \kappa^{2} \sim\left(E_{1,0}^{\mathrm{T}} / E_{\mathrm{C}}\right) l^{-2}$, where $\kappa$ is the inverse size of $T(1,1 ; 0)$, and neglect two last factors in Eq. (7). Using this estimate and substituting $\nu_{\text {eff }}$ for $\nu_{e}-2$ in Eq. (7), we finally get

$$
W_{\mathrm{T}} \sim\left(E_{1,0}^{\mathrm{T}} / \hbar \omega_{c}\right)\left(m_{h} / m_{e}\right)^{2} .
$$

As in Eq. (7), this probability of the radiative decay of a $T(1$, $1 ; 0)$ trion is expressed in units of the $0-0$ transition probability. Note again the presence of the large factor $\left(m_{h} / m_{e}\right)^{2}$; about possible selection rules see Sec. VI.

As can be seen from Fig. 2, there is a clearly visible cyclotron satellite blue shifted approximately $\hbar \omega_{c}$ from the main transition when $\nu_{e}$ exceeds 2 . The integrated intensity of this satellite is roughly a factor of 5 greater than expected for the 1-1 transition, assuming that the number of $n_{h}=1$ holes is given by the Boltzmann factor, and the discrepancy increases as $\nu_{e}$ increases. We tentatively assign this satellite to 1-0 transitions, allowed by the mechanism outlined here.

These two mechanisms of the intrinsic radiative decay of ME's and trions involving $n_{e}=1$ electrons, MP-assisted and scattering-induced decay, are quite general and should operate in any QW containing free electrons. Skolnick et al. ${ }^{42}$ observed two red-shifted satellites, denoted $1_{\mathrm{A}}$ and $1_{\mathrm{L}}$, of the $0-0$ transition in the magnetoluminescence spectra of doped $\mathrm{InGa}_{x} \mathrm{As}_{1-x}$ /InP QW's, as well as blue-shifted bands assigned as $(1,0)$ transitions (Figs. 3 and 8 of their paper). They assigned the red-shifted satellites to phonon replicas of the $(1,0)$ transition. However, this assignment does not explain the spectacular growth in the intensity $I_{\text {sat }}$ of these satellites, relative to that of the $(1,0)$ band $I_{(1,0)}$, when $\nu_{e} \rightarrow 2$ (Fig. 7 in Ref. 42). This phenomenon has been observed in specimens of different quality and must be intrinsic. If one accepts that these red-shifted bands are MP satellites of the sort discussed in Sec. IV while the mechanism of $(1,0)$ transitions is that of this section, Eq. (7), the ratio $I_{(1,0)} / I_{\text {sat }}$ should be proportional to $\left(\nu_{e}-2\right)$ for $0<\nu_{e}-2 \ll 1$. Indeed, for low temperatures the concentration of ME's does not depend on $\nu_{e}$ and is completely controlled by the concentration of photoholes. The factor $\nu_{e}-2$ appears in Eq. (7) through the number of scattering events that is proportional to the number of scatterers, while the scattering cross-section does not depend on either the concentration of ME's or on $\nu_{e}-2$ so long as they are small. This prediction of our theory is confirmed by the data of Ref. 42, as shown in Fig. 3. The spectral position of the satellite $1_{\mathrm{A}}$ fits well the expected position of the charge MP satellite. For example, at $B=15 \mathrm{~T}$ this satellite is red shifted compared to the $0-0$ transition by approximately $10 \mathrm{meV}^{42}$ With $E_{\mathrm{C}} \approx 17 \mathrm{meV}$, this value is about $0.6 E_{\mathrm{C}}$ in agreement with the prediction of the ME theory in the high $B$ limit (see Sec. IV). The red-shifted satellite $1_{\mathrm{L}}$, which is closer to the $0-0$ transition and is seen in a narrower region of magnetic fields (see Fig. 3 of Ref. 42), may be assigned either a spin MP satellite or the new type of trions discussed in Sec. VI below. However, final interpretation of the data needs a more careful study and additional experimental research. 


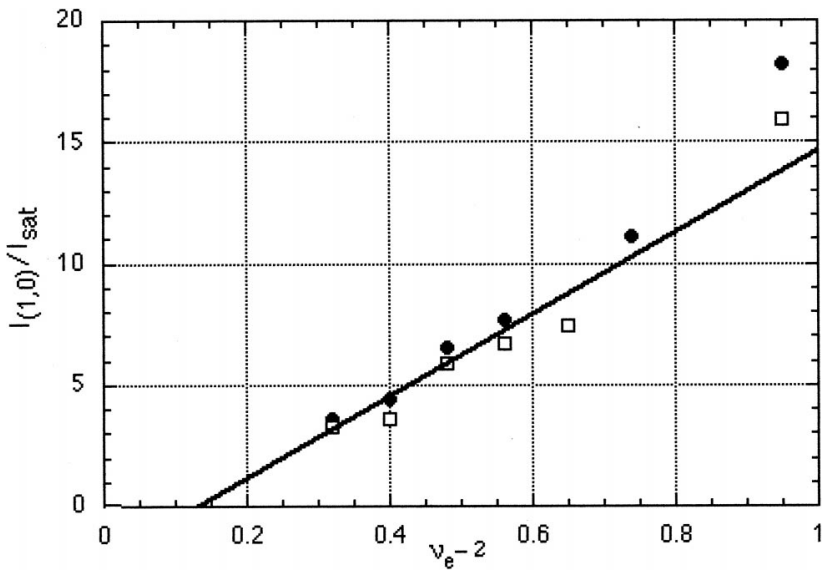

FIG. 3. Intensity ratio of the $(1,0)$ blue-shifted cyclotron satellite to the red-shifted satellite, designated as " $1_{\mathrm{A}}$ " in Ref. 42 and assigned here to a charge MP-assisted transition, against $\nu_{e}-2$. The data points are for two different $\operatorname{In}_{x} \mathrm{Ga}_{1-x}$ As QW's, both containing approximately $9 \times 10^{11}$ electrons per $\mathrm{cm}^{2}$, and are taken from Fig. 7 of Ref. 42 (see Sec. V of the text). While the best straight line through each set of points does not go through the origin, it can be made to do so by assuming that the density given in Ref. 42 is overstated by about $6 \%$, which is well within the error of the measurement.

\section{DO $n_{e}=1$ TRIONS EXIST ?}

In Secs. IV and V, the initial states for emission were (1, 0) ME's, i.e., bound states of one $n_{e}=1$ electron and a $n_{h}$ $=0$ valence hole existing against a background of the completely filled $n_{e}=0 \mathrm{LL}$. The problem that we address here is whether a $n_{h}=0$ hole can bind to two $n_{e}=1$ electrons, forming a trion denoted $T(1,1 ; 0)$. In Secs. IV and $\mathrm{V}$ we have already mentioned that such entities may be initial states for the emission when $\nu_{e}>2$. Nothing is known experimentally about $T(1,1 ; 0)$ trions, and to the best of our knowledge no numerical calculations for such trions have been published. ${ }^{43}$ The problem is important since it concerns the initial state for the emission process. We will argue that $T(1,1 ; 0)$ trions exist and that general arguments suggest that their binding energy $E_{1,0}^{\mathrm{T}}$ is larger rather than smaller than $E_{\mathrm{X}^{-}}$, the binding energy of the usual $n_{e}=0, n_{h}=0$ trions $\mathrm{X}^{-}$. Up to the present time, even the most extensive calculations of $E_{\mathrm{X}^{-}}{ }^{25,44}$ have not reached quantitative agreement with experiment, and calculations of $E_{1,0}^{\mathrm{T}}$ are much more difficult since they should take into account polarization of the $n_{e}$ $=0$ background. Therefore, we use a simplified model suitable for applying general theoretical arguments. Details are given in the Appendix, while here we only summarize basic arguments and results.

For a strong but finite magnetic field, a free electron shows no dispersion while a neutral particle such as a ME is attracted to it by a short-range polarization potential $U_{\mathrm{p}}(r)$ that drops off as $r^{-4}$. If the potential is attractive everywhere, but weak, a trion always exists in 2D. However, its binding energy is exponentially small, $E_{\text {bind }}^{2 \mathrm{D}}$ $\propto \exp \left(-U_{0} /\left|U_{\mathrm{p}}\right|\right),{ }^{45}$ where $U_{0}$ is a characteristic energy of the interaction; cf. Eqs. (A1) and (A2). This estimate is ap- plicable for magnetotrions $X^{-}$since the dispersion law of $(0,0)$ ME's has a minimum at $k=0$. However, the dispersion law of $(1,0)$ magnetoexcitons in $2 \mathrm{D}$ has its minimum at $k_{\text {min }} \neq 0$ [see Eq. (4)]. For this reason, the spectrum near the band bottom is quasi-1D, and binding energy is therefore much larger, being quadratic in $U_{\mathrm{p}}, E_{\text {bind }}^{1 \mathrm{D}} \propto U_{\mathrm{p}}^{2} ;{ }^{45}$ cf. Eqs. (A3) and (A4).

The magnitude of $E_{\text {bind }}^{1 \mathrm{D}}$ is also enhanced by a large factor $m_{h} / m_{e} \gg 1$ and by the fact that the dispersion law of $\operatorname{ME}(1$, 0 ), Eq. (4), is very flat near its minimum. These facts strongly suggest that if the electron-ME interaction is attractive everywhere, $E_{\text {bind }}^{1 \mathrm{D}}$ is larger than $E_{\text {bind }}^{2 \mathrm{D}}$.

However, the problem remains as to whether it is true that the electron-ME interaction is attractive everywhere. When $B=0$, an exciton in 2D (as distinct from 3D) possesses a quadrupole moment whenever the effective masses $m_{e}$ $\neq m_{h}$. For $m_{e}<m_{h}$, the quadrupole electron-ME interaction, $U_{\text {quad }(r) \propto r^{-3}}$, is repulsive for disk-shaped excitons in very narrow quantum wells $w \ll l$ but, because of Gauss's theorem, the interaction changes sign when the QW width $w \sim l$ and becomes attractive for wider QW's when excitons are cylinder shaped. It is shown in Sec. A 2 that in strong fields, i.e., for $\gamma \gg 1$ when LL mixing can be neglected, $\mathbf{k}=0 \mathrm{ME}$ 's have no electric form factor despite the fact that, in the symmetric gauge, electron $n_{e}=1$ quantum states are wider than the $n_{h}=0$ quantum state of the hole with zero angular momentum. In the next order in $1 / B$, and because $k_{\min } \neq 0$, the quadrupole electron-ME interaction appears. Electrostatic arguments suggest that it is attractive for not too narrow QW's, $w \gtrsim l$.

The above arguments are applicable to both singlet and triplet trions and do not say which one is lower in moderate fields. We expect that in the $\gamma \gtrsim 1$ region of interest for us, the singlet $T(1,1 ; 0)$ trion is lower, as in the case of $X^{-}$ trions. $^{25-27}$ Radiative decay of $\mathrm{T}(1,1 ; 0)$ may proceed in different channels, and the spectral shape of the emission depends on the final states. Some of the channels may be prohibited by the selection rules established recently for trions by Dzyubenko and Sivachenko. ${ }^{46}$ They are based on general properties of magnetic symmetry and impose severe restrictions on different types of transitions, especially bound-tobound transitions. ${ }^{47}$ Unfortunately, these selection rules cannot be applied to $T(1,1 ; 0)$ until its internal quantum numbers ( $z$-projection of the angular momentum and the generalized "center-of-orbit,", or "oscillator,', coordinate) are definitely known.

Radiative recombination of $T(1,1 ; 0)$ should be seen as a narrow line if the intraband trion [i.e., a bound state of two $n_{e}=1$ electrons and a single $n_{e}=0$ Fermi hole, which is the two-electron analog of the $\operatorname{MP}(1,0)]$, which we denote $I T(1$, $1 ; 0)$, exists. Since $T(1,1 ; 0)$ and $I T(1,1 ; 0)$ differ only in replacing the $n_{h}=0$ valence hole by the $n_{e}=0$ Fermi-hole, they seem to possess identical symmetry, and, therefore, the bound-to-bound transition must be allowed. This line should be followed by transitions to the two-particle continuum of $n_{e}=1$ electron + MP $(1,0)$ states. At present one cannot exclude the possibility that the narrow $\mathrm{SU}_{0}$ band of Ref. 9 is of the $I T$-assisted type. 
To summarize, the electron-ME attraction due to the exciton polarizability has the same order of magnitude for (1, 0 ) ME's and for $X$ ME's, while the flat quasi-1D dispersion law of $\operatorname{ME}(1,0)$ with $k_{\min } \neq 0$ is especially favorable for forming bound trion states. Therefore, $\mathrm{T}(1,1 ; 0)$ trions are likely to exist, and the above arguments suggest that their binding energy is not small. The exact range of their existence should depend on $\gamma, \epsilon, m_{h} / m_{e}, w / l$, and different parameters. With a completely filled $n_{e}=0$ LL, i.e., $\nu_{e}>2$, these trions are stable. They have no $B=0$ analogs. It would be interesting to look for such trions in the region $\gamma \gtrsim 1$.

\section{VII. $\nu_{E}<2$ REGION}

In this region, the position of the emission band vs $B$ follows the position of the exciton band in the empty crystal rather closely. ${ }^{7}$ This fact is critical for the assignment of the $\nu_{e}=2$ anomaly to the "HS - LL" changeover made in Sec. III, and it indicates that HS is only slightly violated. However, there is no well-established theoretical method for calculating ME spectra in this range of $\nu$ values. Some idea of the qualitative behavior of the spectra can be obtained by analogy with excitons in mixed molecular crystals where lattice sites are randomly occupied by two species. We describe the electron-ME attraction by a pseudo-potential $U(B)<0$, and, for $0<\nu_{e}<1$, we divide all space into occupied (by an electron) and empty "lattice sites" with concentrations $\nu_{e}$ and $1-\nu_{e}$, respectively. Application of the mean-field theory of binary alloys ${ }^{48}$ to this problem suggests that the exciton spectra of the mixed crystal should consist of doublets whose components can be assigned to the specific species constituting the alloy. Note, however, that excitons are collective excitations and, therefore, each exciton state involves both species, the degree of mixing being dependent on the ratio of the exciton bandwidth to the chemical shift [the corresponding ratio in our problem is $E_{\mathbf{C}}(B)$ to $U(B)$ ]. The spectral position of each component of the doublet is a smooth function of the concentrations of the species. However, the most spectacular consequence of this mixing is intensity borrowing that manifests itself as a nonlinear dependence of the intensities of the doublet components on the concentrations of the species; for excitons in binary alloys this nonlinearity is usually rather strong. ${ }^{48}$ Exciton-trion doublets in absorption spectra of QW's have been observed in a number of papers, so it is natural to expect a similar behavior of intensities if the ME's are free. The proper way to investigate this effect would be to measure the ME spectra as a function of $\nu_{e}$ at fixed $B$ [thus keeping $E_{\mathrm{C}}(B)$ and $U(B)$ unchanged]: such a measurement is possible in mixed-type QW's. ${ }^{7}$ Unfortunately, such data are not at present available for GaAs QW's, while the data for CdTe QW's are somewhat confusing. Cox et al. ${ }^{17}$ reported a "triangular" dependence of the oscillator strength of doublet components on electron concentration, which implies the absence of intensity borrowing. On the other hand, Kheng et al. ${ }^{2}$ reported disproportionally strong trion absorption that they attributed to "stolen oscillator strength." Probably, both data were affected by disorder. Data taken from high quality samples are needed.

\section{CONCLUSION}

We conclude that the $\nu_{e}=2$ changeover from Landaulevel-like behavior to trion (or excitonlike) behavior of the magnetophotoluminescence of QW's containing an electron gas is a consequence of hidden symmetry, even though the magnetic field at which this transition is observed is well below the field for which this symmetry may be justified theoretically. For $\nu_{e}>2$, the hidden symmetry is violated and new channels of radiative recombination open: magnetoplasmon-assisted and scattering-induced recombination. They both involve $n_{e}=1$ electrons and the magnetoexciton theory makes specific predictions about the positions and intensities of these spectral bands. The former mechanism accounts for the red-shifted satellite discovered by Finkelstein et $a l .{ }^{9}$ and attributed by them to breaking of hidden symmetry, while the second one accounts for the blueshifted cyclotron satellite. We have shown that there are good reasons to believe that new trions including $n_{e}=1$ electrons and having no analogs in the $B=0$ limit, exist.

\section{ACKNOWLEDGMENTS}

This work was supported by the Department of Energy under Grant No. DEFG 87ER45330.

\section{APPENDIX}

In this appendix we (i) show how the quasi-1D spectrum of ME $(1,0)$ 's contributes to the formation of $T(1,1 ; 0)$ trions, and (ii) prove that $k=0 \mathrm{ME}$ 's formed from different electron and hole LL's, $n_{e} \neq n_{h}$, are completely neutral, i.e., possess no electric form factors in the strong $B$ limit.

\section{Effect of the spectrum dimensionality}

In this section we use polarization interaction as a model for electron-ME attraction in moderately strong magnetic fields and consider a bound state of a ME and a Landau electron. The polarization potential equals $U_{\mathrm{p}}(r) \approx$ $-(\alpha / 2)\left(e / \epsilon r^{2}\right)^{2}$ for $r \geqslant l$ and should be cutoff for $r \leqq l$. Here, $\alpha$ is the ME polarizability, which depends strongly not only on the magnetic but also on the electric field, and, hence, on the distance between the electron and the ME. At large distances $\alpha \approx e^{2}\langle x\rangle^{2} / \hbar \omega_{c} \sim \epsilon l^{3} / \sqrt{\gamma}$. At small distances $r \lesssim l$ the electric field of the electron is large, of the same order as the internal electric field in the exciton. In a strong external electric field, the polarizability $\alpha$ has universal form $\alpha(B)=\left(m_{e}+m_{h}\right) c^{2} / B^{2}$ (Ref. 49) which reduces to $\alpha$ $\sim\left(m_{h} / m_{e}\right) l^{3} / \sqrt{\gamma}$ for $m_{e} \ll m_{h}$. Both estimates result in a common main factor $l^{3} / \sqrt{\gamma}$ multiplied by either $\epsilon$ or $m_{h} / m_{e}$, which are both large. In what follows, we use the second estimate. Near the cutoff point, $r \sim l$, the potential is about $U_{\text {eff }} \sim-\left(m_{h} / m_{e}\right)$ Ry. In the $\gamma \gg 1$ regime, Ry is considered as a small quantity, but because the ratio $m_{h} / m_{e}$ $\approx 5$ in GaAs the potential $U_{\text {eff }}$ is actually not small.

If the potential $U_{\text {eff }}$ is small while the minimum of the ME dispersion law is achieved at $k=0$ (as is the case for $X$ 
formed from $n_{e}=n_{h}=0$ particles), the electron-ME bound state is exponentially shallow. The equation for binding energy in $2 \mathrm{D}$ is ${ }^{45}$

$$
E_{0,0} \approx \frac{\hbar^{2}}{m^{*} l^{2}} \exp \left(-\frac{\hbar^{2} / m^{*}}{\left|\int_{0}^{\infty} U_{\mathrm{p}}(r) r d r\right|}\right),
$$

where $m^{*}$ is the mass of the particle. For a ME $m^{*}$ $\sim \hbar^{2} / E_{\mathrm{C}} l^{2}$, and Eq. (A1) reduces to

$$
E_{\mathrm{X}^{-}} \sim E_{\mathrm{C}} \exp \left(-E_{\mathrm{C}} /\left|U_{\mathrm{eff}}\right|\right) \approx E_{\mathrm{C}} \exp \left(-\beta \gamma^{1 / 2} m_{e} / m_{h}\right)
$$

where $\beta$ is some numerical factor. The last part of Eq. (A2) follows from the observation that $E_{\mathrm{C}}^{2}=2 \hbar \omega_{c} \mathrm{Ry}$. The decrease in the $X^{-}$binding energy with $B$ for $\gamma \geq 1$ is in qualitative agreement with numerical results for singlet trions in narrow QW's. ${ }^{25}$

The problem of a $T(1,1 ; 0)$ trion differs from the above problem in that the dispersion law of $\operatorname{ME}(1,0)$ reaches its minimum at $k_{\min } \neq 0$. In the 2D limit $(w=0), k_{\min } \approx 1.19 / l$, as is discussed in more detail in Sec. IV. Near this minimum, the ME density-of-states shows a typical one-dimensional (1D) singularity, and the problem of shallow bound states near this minimum also becomes quasi-1D. Therefore, the equation for the binding energy is ${ }^{45}$

$$
E_{\mathrm{bind}} \approx \frac{m^{*}}{2 \hbar^{2}}\left[\int_{-\infty}^{\infty} U_{\mathrm{p}}(x) d x\right]^{2} .
$$

For $m^{*}$ we will use the same estimate $m^{*} \sim \hbar^{2} / E_{\mathrm{C}} l^{2}$ as above. However, the numerical value of $m^{*}$ for $\operatorname{ME}(1,0)$ is larger than for $X$ because $E_{1,0}^{\mathrm{ME}}(k)$ is flat near its minimum. Finally

$$
E_{1,0}^{\mathrm{T}} \sim U_{\mathrm{eff}}^{2} / E_{\mathrm{C}} \approx \beta_{1}\left(E_{\mathrm{C}} / \gamma\right)\left(m_{h} / m_{e}\right)^{2},
$$

where $\beta_{1}$ is a numerical factor. Unlike Eq. (A2), Eq. (A4) does not include any factor that can be exponentially small. Therefore, it implies that $T(1,1 ; 0)$ trions exist, and provides a good reason to expect that their binding energy is not less than that of $X^{-} .50$

Note that a large value of $m_{h} / m_{e}$ strongly enhances the trion binding energy both in 2D and in quasi-1D regimes. This statement is in agreement with the fact that while the singlet $X^{-}$does not exist in the lowest LL approximation while the $D^{-}$singlet, a negative donor state, does exist. ${ }^{25}$

The derivation of Eqs. (A3) and (A4) was based on the assumption that the electron-ME interaction is attractive as the polarization interaction always is. This assumption is not obvious for $T(1,1 ; 0)$ since $(1,0)$ ME's possess dipole moments $\mathbf{d} \neq 0$ near $k_{\min } \neq 0$ where the minimum of the ME (1, $0)$ energy spectrum is reached, cf. Sec. IV. The dipole moment is $\mathbf{d}(\mathbf{k})=(\hat{\mathbf{z}} \times \mathbf{k}) l^{2}$, where $\hat{\mathbf{z}}$ is the unit vector perpendicular to the confinement plane. Avoiding details, we only mention here that in the bound-state problem the dipole moments of the in- and out-going magnetoexciton waves have opposite signs. Since these waves enter with equal weights into the bound-state wave function, contributions from the static dipoles cancel, and the effect of these dipoles on shallow bound states is minor.

For both the trion binding energies evaluated above, $E_{\mathrm{X}^{-}}$ and $E_{1,0}^{\mathrm{T}}$, the ratios $E_{\mathrm{X}^{-}} / E_{\mathrm{C}}$ and $E_{1,0}^{\mathrm{T}} / E_{\mathrm{C}}$ tend to zero as $B$ $\rightarrow \infty$ since the polarizability $\alpha(B) \rightarrow 0$ in this limit. However, triplet trions $X^{-}$remain bound in this limit since electronic correlations rather than polarizabilities become the main factor. $22,24,25$

\section{Asymptotic neutrality of magnetoexcitons}

We prove in this section that in the $\gamma \rightarrow \infty$ limit, $\mathbf{k}=02 \mathrm{D}$ ME's are completely neutral particles even for $n_{e} \neq n_{h}$, i.e., they have no Coulomb form factors and therefore cannot produce any repulsive Hartree potential for electrons.

The wave functions of ME's with quantum numbers $\mathbf{k}, n_{e}$, and $n_{h}$ are completely determined by the condition of translational symmetry as

$$
\begin{aligned}
\Phi_{\mathbf{k} n_{e} n_{h}}\left(\mathbf{r}_{e}, \mathbf{r}_{h}\right)= & (2 \pi / S)^{1 / 2} \sum_{p} \psi_{n_{e} k_{y} / 2+p}\left(\mathbf{r}_{e}\right) \psi_{n_{h} k_{y} / 2-p}\left(\mathbf{r}_{h}\right) \\
& \times \exp \left(-i k_{x} p\right),
\end{aligned}
$$

where $\psi_{n p}(\mathbf{r})$ are electron wave functions in the Landau gauge. After the transformation to the new variables $\mathbf{r}=\mathbf{r}_{e}$ $-\mathbf{r}_{h}$ and $\mathbf{R}=\left(\mathbf{r}_{e}+\mathbf{r}_{h}\right) / 2$ and integrating over $p$ in Eq. (A5), functions $\Phi_{\mathbf{k} n_{e} n_{h}}\left(\mathbf{r}_{e}, \mathbf{r}_{h}\right)$ take the form

$$
\Psi_{\mathbf{k} n_{e} n_{h}}(\mathbf{r}, \mathbf{R})=(2 \pi / S)^{1 / 2} J_{n_{e} n_{h}}(\mathbf{r}-\mathbf{d}) \exp [-i X y+i(\mathbf{k} \cdot \mathbf{R})] .
$$

Here, functions $J_{m n}(\mathbf{r})$, as in Eq. (5) of Ref. 28, are

$$
\begin{aligned}
& J_{m n}(\mathbf{r})=(n ! / m !)^{1 / 2} \exp \left(-r^{2} / 4\right) \\
& \times[(i x+y) / \sqrt{2}]^{m-n} L_{n}^{m-n}\left(r^{2} / 2\right), \\
& m>n,
\end{aligned}
$$

where $L_{n}^{m}$ are Laguerre polynomials, and $J_{m n}(\mathbf{r})=J_{n m}^{*}(-\mathbf{r})$.

The choice of center-of-exciton coordinate $\mathbf{R}$ is uniquely determined by the condition that the functions $J_{n_{e} n_{h}}(\mathbf{r}-\mathbf{d})$ are eigenfunctions of the angular momentum for the variable $\mathbf{r}-\mathbf{d}$. The physical meaning of $\mathbf{d}$ as the ME dipole moment is confirmed by the expression for the mean value of $\mathbf{r},\langle\mathbf{r}\rangle$ $=\mathbf{d}$, which follows from Eq. (A6).

The function $\Psi_{\mathbf{k} e_{e} n_{h}}(\mathbf{r}, \mathbf{R})$ possesses the property

$$
\left|\Psi_{\mathbf{k}=0 n_{e} n_{h}}(\mathbf{r}, \mathbf{R})\right|^{2}=\left|\Psi_{\mathbf{k}=0 n_{e} n_{h}}(-\mathbf{r}, \mathbf{R})\right|^{2},
$$

and, therefore, the exciton charge density in the reference frame in which the center-of-exciton coordinate $\mathbf{R}$ is at rest in the origin, $\mathbf{R}=0$, is

$$
\begin{aligned}
\rho_{\mathbf{k}=0 n_{e} n_{h}}(\mathbf{r})= & \iint d \mathbf{r}_{e} d \mathbf{r}_{h}\left|\Phi_{\mathbf{k}=0 n_{e} n_{h}}\left(\mathbf{r}_{e}, \mathbf{r}_{h}\right)\right|^{2}\left[\delta\left(\mathbf{r}_{h}-\mathbf{r}\right)\right. \\
& \left.-\delta\left(\mathbf{r}_{e}-\mathbf{r}\right)\right] \delta\left(\mathbf{r}_{e}+\mathbf{r}_{h}\right) \\
= & \left|\Psi_{\mathbf{k}=0 n_{e} n_{h}}(-2 \mathbf{r}, \mathbf{R}=0)\right|^{2}
\end{aligned}
$$




$$
-\left|\Psi_{\mathbf{k}=0 n_{e} n_{h}}(2 \mathbf{r}, \mathbf{R}=0)\right|^{2}=0
$$

everywhere. This property is counter intuitive for $n_{e} \neq n_{h}$ since the free electron and hole wave functions are very different. It comes from the fact that, in the absence of the electron and hole kinetic energy, the minimum of the total energy is also the minimum of the electrostatic energy, which, for $\mathbf{k}=0$ and therefore $\mathbf{d}=0$, is reached for a neutral system.

The role of the form factors that arise when $\mathbf{k} \neq 0$ is discussed in Secs. VI and A 1.
*Email: erashba@ hotmail.com

${ }^{1}$ C. H. Perry, J. M. Worlock, M. C. Smith, and A. Petrou, in High Magnetic Fields in Semiconductor Physics, edited by G. Landwehr (Springer, Berlin, 1987), p. 202.

${ }^{2}$ K. Kheng, R. T. Cox, Y. M. d'Aubigné, F. Bassani, K. Saminadayar, and S. Tatarenko, Phys. Rev. Lett. 71, 1752 (1993).

${ }^{3}$ A. J. Shields, M. Pepper, D. A. Ritchie, M. Y. Simmons, and G. A. C. Jones, Phys. Rev. B 51, 18049 (1995).

${ }^{4}$ G. Finkelstein, H. Shtrikman, and I. Bar-Joseph, Phys. Rev. Lett. 74, 976 (1995).

${ }^{5}$ D. Gakhtman, E. Cohen, A. Ron, and L. N. Pfeiffer, Phys. Rev. B 54, 10320 (1996).

${ }^{6}$ S. R. Ryu, W.-U. Yu, L. P. Fu, Z. H. Jiang, A. Petrou, B. D. McCombe, and W. Schaff, Surf. Sci. 361-362, 363 (1996).

${ }^{7}$ H. W. Yoon, M. D. Sturge, and L. N. Pfeiffer, Solid State Commun. 104, 287 (1997).

8 This changeover is often called, rather inaccurately, a "Mott transition." The mechanism driving this changeover has nothing to do with that driving the true Mott transition, and it would be better named a "symmetry-driven transition."

${ }^{9}$ G. Finkelstein, H. Shtrikman, and I. Bar-Joseph, Phys. Rev. B 56, 10326 (1997).

${ }^{10}$ I. V. Lerner and Yu. E. Lozovik, Zh. Éksp. Teor. Fiz. 80, 1488 (1981) [Sov. Phys. JETP 53, 763 (1981)].

${ }^{11}$ Yu. A. Bychkov, S. V. Iordanskii, and G. M. Eliashberg, Poverkhnost 1982 (10), 33 (1982).

${ }^{12}$ A. H. MacDonald and E. H. Rezayi, Phys. Rev. B 42, 3224 (1990).

${ }^{13}$ A. B. Dzyubenko and Yu. E. Lozovik, J. Phys. A 24, 414 (1991).

${ }^{14}$ V. M. Apal'kov and E. I. Rashba, Pis'ma Zh. Éksp. Teor. Fiz. 53, 148 (1991) [JETP Lett. 53, 155 (1991)].

${ }^{15}$ E. I. Rashba and J. L. Birman, Solid State Commun. 84, 99 (1992)

${ }^{16}$ E. I. Rashba, M. D. Sturge, H. W. Yoon, and L. N. Pfeiffer, Solid State Commun. 114, 593 (2000).

${ }^{17}$ R. T. Cox, V. Huard, K. Kheng, S. Losiva, R. B. Miller, K. Saminadayar, A. Arnoult, J. Cibert, S. Tatarenko, and M. Potemskii, Acta Phys. Pol. A 94, 99 (1998).

18 Hidden symmetry is an exact dynamic symmetry that takes into account electron correlations. For neutral electron-hole plasmas with $\nu_{e}=\nu_{h}$ slightly larger than $2 N$ or slightly smaller than $2(N+1)$ this symmetry can be understood in simple terms. The energy of the Coulomb electron-hole attraction in an ME, in the former system, is exactly equal to the additional exchange energy per electron, in the latter system (Ref. 28).

${ }^{19}$ Yu. A. Bychkov and E. I. Rashba, Zh. Éksp. Teor. Fiz. 85, 1826 (1983) [Sov. Phys. JETP 58, 1062 (1983)].

${ }^{20}$ D. Paquet, T. M. Rice, and K. Ueda, Phys. Rev. B 32, 5208 (1985).

${ }^{21}$ B. A. Muzykantskii, Zh. Éksp. Teor. Fiz. 101, 1084 (1992) [ Sov. Phys. JETP 74, 897 (1992)].
${ }^{22}$ A. Wojs and P. Hawrylak, Phys. Rev. B 51, 10880 (1995).

${ }^{23}$ M. Bayer, O. Stern, P. Hawrylak, S. Fafard, and A. Forchel, Nature (London) 405, 923 (2000).

${ }^{24}$ J. J. Palacios, D. Yoshioka, and A. H. MacDonald, Phys. Rev. B 54, R2296 (1996).

${ }^{25}$ D. M. Whittaker and A. J. Shields, Phys. Rev. B 56, 15185 (1997) and references therein.

${ }^{26}$ F. M. Munteanu, Y. Kim, C. H. Perry, D. G. Rickel, J. A. Simmons, and J. L. Reno, Phys. Rev. B 61, 4731 (2000) and references therein.

${ }^{27}$ A. Wojs, J. J. Quinn, and P. Hawrylak, cond-mat/0001327 (unpublished).

${ }^{28}$ Yu. A. Bychkov and E. I. Rashba, Phys. Rev. B 44, 6112 (1991).

${ }^{29}$ L. V. Butov, V. D. Kulakovskii, and E. I. Rashba, Pis'ma Zh. Éksp. Teor. Fiz. 53, 104 (1991) [ JETP Lett. 53, 109 (1991)].

${ }^{30}$ L. V. Butov and V. D. Kulakovskii, Pis'ma Zh. Éksp. Teor. Fiz. 53, 444 (1991) [ JETP Lett. 53, 466 (1991)].

${ }^{31}$ Yu. A. Bychkov, S. V. Iordanskii, and G. M. Eliashberg, Pis'ma Zh. Éksp. Teor. Fiz. 33, 152 (1981) [ JETP Lett. 33, 143 (1981)].

${ }^{32}$ C. Kallin and B. I. Halperin, Phys. Rev. B 30, 5655 (1984).

${ }^{33}$ A. H. MacDonald, J. Phys. C 18, 1003 (1984).

${ }^{34}$ M. J. Manfra, B. B. Goldberg, L. Pfeiffer, and K. West, Phys. Rev. B 57, R9467 (1998).

${ }^{35}$ M. Hayne, C. L. Jones, R. Bogaerts, A. Usher, F. Herlach, V. V. Moshchalkov, and M. Henini, Physica B 256-258, 327 (1998).

36 Spin indices of valence holes in Eq. (2) and in what follows are chosen symbolically to simplify equations. Final results do not depend on this simplification.

${ }^{37}$ It is much more difficult to compare the radiative lifetimes and relative photoluminescence intensities of these processes since the lifetime of a $\operatorname{ME}(0,0)$ depends on its coherence length, which is controlled by localization phenomena, all kinds of scattering mechanisms, etc.

${ }^{38}$ W. Kohn, Phys. Rev. 123, 1242 (1961).

39 Therefore, this satellite is not related to any shake-up process. We have retained the original designation of it as $\mathrm{SU}_{0}$, which might be taken to imply such a relation, only to make direct connection to the experimental results of Ref. 9.

40 The system investigated in Refs. 7 and 16 is a superlattice rather than an isolated QW. Interaction of QW's can be neglected for interband ME's but not for MP's. For them the resonance term $H_{\mathrm{r}}$ in the Hamiltonian acquires an inter-QW contribution that is of the order of $\exp (-k b)$, where $k \sim 1 / l$ is a typical momentum and $b$ is the separation between QW's. This contribution can change the shape of the MP satellite, but in the sample used in Refs. 7 and 16 it was only about 0.15 .

${ }^{41}$ J. F. Janak, Phys. Rev. 178, 1416 (1969).

${ }^{42}$ M. S. Skolnick, K. J. Nash, M. K. Saker, and S. J. Bass, Phys. Rev. B 50, 11771 (1994). 
${ }^{43}$ Very recently trions $T(1,0 ; 0)$ were found by computations in the strong $B$ region (Ref. 46).

${ }^{44}$ A. Moradi and B. Stébé, Solid State Commun. 112, 499 (1999).

45 L. D. Landau and E. M. Lifshitz, Quantum Mechanics: Nonrelativistic Theory (Pergamon, New York, 1977), Sec. 45.

${ }^{46}$ A. B. Dzyubenko and A. Yu. Sivachenko, Phys. Rev. Lett. 84, 4429 (2000).

${ }^{47}$ A. B. Dzyubenko, A. Yu. Sivachenko, H. A. Nickel, T. M. Yeo,
G. Kioseoglou, B. D. McCombe, and A. Petrou, Physica E (Amsterdam) 6, 156 (2000).

48 V. L. Broude, E. I. Rashba, and E. F. Sheka, Spectroscopy of Molecular Excitons (Springer, Berlin, 1985), Chap. 4.

${ }^{49}$ L. P. Gor'kov and I. E. Dzyaloshinskii, Zh. Éksp. Teor. Fiz. 53, 296 (1968) [ Sov. Phys. JETP 26, 449 (1968)].

${ }^{50}$ Unfortunately, we cannot evaluate numerical values of $\beta$ and $\beta_{1}$ because they depend strongly on cutoff radius. 